\title{
L'enjeu primordial du divorce : les enfants
}

\author{
Alain TRANNOY*
}

Joseph Stiglitz a l'habitude de dire en plaisantant que le « seul vrai choix » sur lequel il ne faut pas se tromper dans la vie est le choix de ses parents. Alors « faire le choix » de naître dans un foyer dont les parents divorcent, est-ce réellement un mauvais choix ? Cet aspect relativement peu exploré dans l'article semble nécessiter des développements complémentaires, après avoir indiqué en accord avec les auteures que, s'agissant des parents, c'est en grande partie leur responsabilité d'avoir choisi un partenaire avec lequel ils n'ont pas réussi à s'entendre.

Le divorce peut s'interpréter d'une manière schématique comme une inadéquation dans la procédure d'appariement qui, dans les sociétés occidentales ${ }^{(1)}$, ne repose que sur les épaules des individus et ne fait plus intervenir la famille, comme c'était le cas dans les sociétés traditionnelles. Cette inadéquation peut résulter d'une mauvaise adaptation à des évènements fortuits (mobilité professionnelle ascendante ou descendante de l'un des conjoints par exemple) ou à une mauvaise prise en compte de certaines difficultés de la vie commune. Toutefois, les auteures ne mentionnent pas, même si tout ceci résulte de décisions prises par les individus, le fait qu'il peut exister un certain déterminisme social dans la décision de divorcer, qui résulterait spécifiquement d'une influence du divorce parental sur le divorce filial, ou d'une corrélation intergénérationnelle entre des caractéristiques peu favorables du milieu d'origine et la probabilité d'un divorce dans la population des descendants.

Les enfants de divorcés divorcent-ils plus par exemple? La fragilité relationnelle ou le mauvais appariement peuvent-ils faire l'objet d'une transmission intergénérationnelle? Si tel est le cas, et ce point n'est pas documenté dans l'article, le raisonnement de John Roemer (1998) s'applique selon nous (voir aussi Roemer et Trannoy, 2015). Celui-ci oppose « les circonstances » qui regroupent des facteurs d'influence qui s'imposent à l'individu comme le milieu social ou les aléas à "l'effort ", terme générique pour désigner des facteurs dont l'individu peut être tenu pour responsable. Une différence de choix sys-

(1) Il ne s'agit en aucun cas d'un jugement de valeur sur le fait de savoir si la procédure d'appariement dans les sociétés occidentales est meilleure que celle qui avait cours dans les sociétés traditionnelles.

* Aix-Marseille Université (AMSE), CNRS, EHESS. 
tématique en moyenne est une circonstance, une caractéristique indépendante des individus. Comme toute circonstance, elle doit faire l'objet d'une politique compensatoire de la part des pouvoirs publics, afin de rétablir l'égalité des chances. Quelle forme peut-elle prendre? L'habitude de pensée conduit à préconiser une redistribution monétaire, alors qu'une vision d'égalité des chances privilégie une politique préventive plutôt qu'une politique curative, du moins si l'on privilégie une approche ex ante à une approche ex post. Dans ce cas, on peut penser à des bons (vouchers) offrant une réduction chez des conseillers conjugaux, des psychologues, ou des psychiatres, même si le recours à un conseiller conjugal du planning familial est déjà gratuit. En l'absence d'enfants, la politique compensatoire entre ex-époux est du domaine privé. A priori, la négociation de la compensation entre les deux ex-époux est susceptible d'une analyse coasienne, (Coase, 1960), l'individu désirant la rupture devant compenser l'autre individu, à moins que d'un commun accord les deux individus veuillent reprendre leur liberté et envisagent des prestations compensatoires en fonction des investissements spécifiques consentis dans le couple. L'auteur analyse avec justesse le déséquilibre entre les deux sexes. Dans la majorité des cas, les hommes ont plus investi sur le marché du travail dont les rendements a priori sont indépendants de la situation matrimoniale, même si de nombreux travaux mettent en évidence une prime salariale au mariage qui peut être dûe à un effet de sélection ou à un plus grand investissement. Les femmes, en particulier celles qui ont abandonné leur travail pour s'occuper de l'éducation des enfants, ont en général plus investi dans des activités domestiques dont le rendement est spécifique à la situation matrimoniale. Lanalyse pourrait être étendue au régime de retraite. Dans un régime de retraite par capitalisation, le capital accumulé par les deux conjoints pourrait être divisé par deux, tout comme le patrimoine acquis durant la communauté. Le régime de retraite par répartition par points de type suédois pourrait aussi faire l'objet d'un partage en deux du capital de points accumulés par les conjoints. La gestion du système de retraite par répartition en France, qui n'est pas unifié et dont les droits ne s'ouvrent qu'à la clôture, n'est malheureusement pas adapté à cette solution de partage. Le système de retraite par répartition peut être aussi une source d'inégalités au moment de la séparation s'il incite les individus à trop peu épargner pour leurs vieux jours. Le patrimoine accumulé au moment de la séparation et susceptible d'un partage est alors plus faible que dans un régime de capitalisation. Cet argument dans le cas de la France doit être relativisé puisque le taux d'épargne en population générale reste aux alentours de 14,5 \% en 2015 selon l'Insee, un taux élevé au regard des comparaisons internationales.

Si les parents sont responsables d'un divorce, les enfants le subissent. Et pour ces derniers, il s'agit clairement d'une circonstance, du moins si être enfant de divorcé constitue un handicap dans le parcours éducatif et par conséquent sur le marché du travail, ainsi que dans les relations sociales et privées. Le conflit classique entre le principe de responsabilité et le principe de compensation trouve là matière à s'appliquer. Si l'on privilégie le principe 
de récompense naturelle qui énonce que les personnes ne doivent pas être compensées pour les facteurs dont ils peuvent être tenus responsables, les parents doivent subir les conséquences de leur décision de divorcer. Comme les enfants ont partie liée avec leurs parents, au moins jusqu'à leur majorité, aucune compensation du handicap n'est consentie. Si, en revanche, priorité est donnée au principe de compensation, alors la politique publique doit aménager des dispositions permettant aux enfants de divorcés de surmonter leur handicap. La politique sociale en France privilégie nettement le principe de récompense naturelle. Il suffit de comparer le traitement fiscal au titre de l'impôt sur le revenu des divorcés et des veufs pour ceux qui ont encore des enfants à charge. Les divorcés ne bénéficient d'aucune réduction spécifique et ont le droit, comme tous les parents isolés qui élèvent seuls un enfant à charge, à une demi-part supplémentaire. En revanche, les veufs et veuves qui élèvent toujours des enfants bénéficient du même nombre de parts que les couples mariés ou pacsés ayant des enfants à charge, soit 2,5 parts par exemple pour une veuve avec un enfant à charge (avantage plafonné à $4672 €$ en 2014). Ce traitement différencié est intéressant dans la mesure où, même si Marc Fleurbaey et François Maniquet (2012) considèrent que l'on peut donner priorité à l'un des principes plutôt qu'à un autre, l'inclinaison est plutôt, parmi les philosophes et les économistes travaillant sur le concept de l'égalité des chances, de donner priorité au principe de compensation. Supposons que l'on suive cette inclinaison. La question centrale est alors de savoir si on peut dans une certaine mesure cibler les enfants plutôt que les parents. C'est un impensé de la politique sociale. La seule disposition à l'œuvre dans l'arsenal fiscalo-social qui cible d'une manière indirecte les parents divorcés est la demi-part pour parent isolé (veufs ou divorcés) : la demi-part concerne des personnes qui vivent seules et ont élevé seules pendant au moins 5 ans un enfant qui n'est plus à leur charge. Avant 2014, le seuil des 5 ans n'était pas requis pour bénéficier de cette disposition qui réduit l'impôt sur le revenu à payer dans la limite d'un plafond fixé à $897 €$ cette année-là, une réduction somme toute modeste. Les fondements de cette disposition sont loin d'être évidents au regard de la théorie de l'égalité des opportunités. Une compensation est accordée aux parents pourtant responsables du divorce, et c'est a priori contraire au principe de responsabilité. Cette compensation ne peut aider les enfants qu'indirectement, d'autant que les enfants sont déjà élevés. Une préconisation directement en accord avec le principe de compensation serait d'aider les enfants en ciblant des biens qui les concernent à travers une politique de bons (vouchers) : essentiellement le confort et la qualité du logement, les cours particuliers, les vacances. Cette politique sera accusée d'être paternaliste, et elle l'est assurément puisqu'il s'agit d'aider les enfants sans trop aider les parents. La déclinaison de cette politique lorsque l'enfant est devenu adulte a déjà été évoquée au début de ce commentaire et constituerait le second volet d'une politique tendant à assurer à l'enfant de divorcés une plus grande chance de réussir sa vie d'adulte. 


\section{RÉFÉRENCES}

COASE R., 1960, « The problem of social cost », Journal of Law and Economics, 3(1), p. $1-44$.

FLEURBAEY M., MANIQUET F., 2012, Equality of Opportunity. The Economics of Responsibility, World Scientific Publishing, 332 p.

RoEMER J., 1998, Equality of Opportunity, Harvard University Press, 128 p.

ROEMERJ., TRANNOY A., 2015, « Equality of Opportunity » in Atkinson A., Bourguignon F. (eds), Handbook of Income Inequalities, Vol. 2A, p. 217-300. 